\title{
RUMANÍA EN EL CONTEXTO GEOPOLÍTICO DEL MAR NEGRO
}

\author{
Silvia Marcu* \\ Doctora en Geografía Humana; Instituto de Economía y Geografía del CSIC. \\ C/Pinar, 25; 28006 Madrid
}

\begin{abstract}
"La transformación del único vecino amigo de Rumania, el Mar Negro, en un nuevo pivote geopolítico es una oportunidad, casi ínica. En estos momentos cruciales de nuestra bistoria, tenemos la ocasión de velar al mismo tiempo por los intereses del espacio rumano y por los del espacio euro-atlántico, sobrepuestos de manera ejemplar, como un cifro olvidado y reinventado perfectamente con el mismo cúmulo de valores". (Victor Roncea, 2005).
\end{abstract}

Resumen: El artículo se propone abordar cuál es la posición actual que tiene Rumanía, país europeo, balcánico, cárpato, danubiano y póntico, en la región geopolítica del Mar Negro, como miembro de la Alianza Atlántica y en vísperas de su ingreso en la UE.

La primera parte presenta el marco actual de la región geopolítica del Mar Negro, para que, a continuación se analicen las dimensiones geopolíticas rumanas, su nueva estrategia y las prioridades de actuación en la zona, destacando la influencia americana y la reciente propuesta del país de constituir un partenariado estratégico Washington-Londres-Bucarest. La tercera parte analiza las implicaciones rumanas en las tensiones de los países de la cuenca del Mar Negro, ejemplificando los casos de Transdniester, la isla de las Serpientes y la construcción por parte de Ucrania, del canal Bastroe en el delta del Danubio.

Finalmente, se analiza la gravitación de Rumanía en el sistema geopolítico del Mar Negro en el siglo XXI.

Palabras clave: Rumanía, geopolítica, Mar Negro, estrategia, región.

Abstract: The article sets out to approach which is the present position that has Rumanía, European, Balkan, Carpathians, Danube, and Pontiac's country, in the geopolitical region of the Black Sea, like member of the Atlantic Alliance and on the eve of its entrance in the UE.

* Recibido: 11-5-2006. Aceptado: 22-11-2006 
The first part display the present frame of the geopolitical region of the Black Sea, so that, next the Rumanian geopolitical dimensions, their new strategy and the priorities of performance in the zone are analyzed, emphasizing the American influence and the recent proposal of the country to constitute strategic partnership Washington-London-Bucharest. The third part analyzes the Rumanian implications in the tensions of the countries of the river basin of the Black Sea, exemplifying the cases of Transdniester, the island of the Serpents and the construction on the part of the Ukraine, of the Bastroe arm in the delta of the Danube.

Finally, the gravitation of Rumanía in the geopolitical system of the Black Sea of century XXI is analyzed.

Key word: Romania, geopolitics, the Black Sea, strategy, region.

\section{Una nueva identidad para el Mar Negro}

La geopolítica de la región del Mar Negro sufrió cambios importantes desde el desplome de la Unión Soviética. A pesar de que la Federación Rusa mantiene vínculos económicos con los países de la región, en los últimos años comenzó a manifestarse una nueva dinámica geopolítica, claramente orientada hacia Occidente, a través de la intervención americana y de la participación más activa de la Unión Europea (UE).

Aunque muy lentamente, los acontecimientos de relevante importancia de los últimos años llamaron la atención a Occidente sobre el Mar Negro - región geopolítica que incluye los países con salida al mar, Moldavia y los Estados del Cáucaso de Sur: Armenia, Azerbaiyán y Georgia. El anclaje y la integración en la Comunidad euroatlántica, llevada a cabo con éxito (en 2004) por los países de la Europa Central y Oriental, desde el Mar Báltico hasta el Mar Negro, marcan el final del ambicioso y original proyecto geopolítico de los años 90, iniciado a finales de la Guerra Fría.

Los ataques terroristas del 11 de septiembre de 2001, del 11 de marzo de 2003 y de 7 de julio de 2005 contribuyeron a subrayar los peligros del nuevo siglo y pusieron de manifiesto el hecho de que las mayores amenazas para América del Norte y para Europa pueden llegar descle el exterior de los dos continentes. Estos acontecimientos trajeron a la región geopolítica del Mar Negro en el centro de la atención occidental. Al mismo tiempo, los acontecimientos revelaron el hecho de que el Occidente no tiene aún una estrategia coherente para esta región (Asmus y Jakson, 2004). 


\section{1. ¿Qué es la región del Mar Negro?}

La historia de la región del Mar Negro que el paso del tiempo ha convertido en clave y síntesis de Asia y Europa, de Oriente y Occidente, de Cristianismo e Islam, ha venido a confirmar en el presente su carácter de encrucijada estratégica de culturas, religiones y pueblos diversos. Quizá haya sido la atracción que despiertan sus inmensas reservas naturales, o su valor geopolítico, o el empeño de la historia en redibujar sus fronteras interiores durante siglos; lo cierto es que todos los imperios que han ido desfilando por la región la han convertido en escenario de batallas y luchas por el poder: desde las legendarias Grecia, Roma y Bizancio, pasando por el dominio mongol, hasta llegar a los imperios persa, otomano y ruso; estos tres últimos, son los que, de forma más decisiva, han configurado la historia reciente de la región y dejado un legado que todavía hoy, en los comienzos del siglo XXI, parece imposible de olvidar.

Geopolíticamente, el Mar Negro se encuentra en la confluencia de los tres antiguos imperios ya mencionados: zarista, persa y otomano. Durante la época de la Guerra Fría la región sufrió una separación adicional entre el Este y el Oeste. Las dos "revoluciones" que afectaron a la zona, la de 1989 y la de 1991, llevaron al desplome de las dictaduras en la Europa del Este y a la disolución de la Unión Soviética. Estos acontecimientos representan, por primera vez desde el siglo XIX, un nuevo capítulo abierto en la historia de la región. Bulgaria, Rumanía y Turquía, en calidad de miembros de la Alianza Atlántica OTAN que dominan las fronteras del sur y las del oeste, junto a los nuevos Estados independientes de la CEI (Comunidad de Estados Independientes) Moldavia, Ucrania, Rusia y Georgia en las fronteras nórdicas y orientales, empiezan a conferir una forma característica a esta región. (Ionescu, 2005). El Mar Negro comprende, asimismo, los Estados del Cáucaso - además de Georgia, Armenia y Azerbaiyán y, por tanto, el corredor energético euro-asiático que une el sistema euro-atlántico con los recursos energéticos de la zona del Mar Caspio y de los Estados de Asia Central. Además, se pueden emitir algunas afirmaciones en relación con la proyección de un sistema estable del Mar Negro al norte de Transdniester, Odessa y Sujumi, puesto que un sistema de estas características supone la solución de los conflictos situados a lo largo del arco noréstico y el acceso a los grandes ríos comerciales que desembocan en el Mar Negro: el Danubio, el Dniéster y el Dnieper.

En 1992 Turquía propuso la creación de una cooperación regional en la región, bajo el nombre de la Cooperación Económica del Mar Negro (CEMN). A pesar de ser un sistema regional de cooperación, junto a GUAAM (Georgia, Ucrania, Armenia, Azerbaiyán Moldavia), un mecanismo de coordinación entre algunas ex repúblicas soviéticas, la CEMN llevó a un aumento del interés común al nivel político y económico. La formulación de la política de "Dimensión del Sur" de la seguridad europea del 2001 y la adhesión de Rumanía y de Bulgaria a la OTAN en abril de 2004 representan una confirmación del hecho de que estos países, junto a Turquía, son socios 
de un sistema único de seguridad que, a su vez, está plenamente integrado en el amplio sistema euro-atlántico. Actualmente, tanto Ucrania como Georgia, países que registraron cambios pro-occidentales importantes a comienzos del siglo XXI (2003, "la revolución de las rosas" en Georgia, y 2004, "la revolución naranja" en Ucrania) persiguen su ingreso en la OTAN, hecho que sugiere que los dos miran su futuro en términos de una seguridad y cooperación común en el Mar Negro.

Una convergencia similar de intereses regionales se puede vislumbrar en el desarrollo de las relaciones con la UE: además del próximo ingreso de Rumanía y Bulgaria previsto para 2007, y del comienzo de las negociaciones de Turquía con la UE, destacamos la decisión de ésta de extender su Política de Vecindad hacia Georgia, Azerbaiyán y Armenia. De esta manera, todos los Estados, inclusive Rusia, Ucrania y Moldavia, se implicarán en relaciones mucho más estrechas con la UE. Los intereses económicos y de seguridad común y la atracción gravitacional de una Europa de integración rápida empujan a los Estados del Mar Negro hacia una convergencia regional. Y, mientras la persistencia de los conflictos latentes y la fragilidad de las instituciones nacionales sugieren que la aparición de un sistema geopolítico funcional en la zona tardará en estabilizarse, existen claros indicios de la conversión de la región en una zona unitaria.

En la actualidad, el mapa político del Mar Negro está influenciado por una ex súper potencia, Rusia, y por dos potencias regionales, Ucrania y Turquía. Como sucesora de la Unión Soviética, Rusia representa un actor importante en la región. La concepción neo-imperialista de Rusia en el Mar Negro tiene su origen en las épocas zarista y soviética pero sobre todo en la necesidad de oponerse a la expansión de Estados Unidos y de la UE en la región. En este sentido, el presidente ruso Vladimir Putin manifestó un interés permanente para la flota rusa del Mar Negro que a pesar de haber disminuido, representa aún una fuerza significativa ${ }^{1}$. Las declaraciones del presidente ruso del tipo "... la cuenca del Mar Negro y del mar de Azov se encuentra en la zona rusa de interés estratégico" o "el Mar Negro ofrece a Rusia acceso directo a las más importantes rutas globales de transporte"2 subrayan el hecho de que Rusia no desea abandonar sus posiciones en el Mar Negro. Rusia sigue manteniendo sus bases militares en Georgia (Batumi y Akhalkalaki), a pesar del compromiso adquirido en la cumbre de la OSCE (Organización para la Seguridad y Cooperación en Europa) en 1999, en Estambul. Al mismo tiempo explota las tendencias separatistas de Abjazia y de Osetia del Sur y de Adzaria (Asmus, Dimitrov, Forbrig, 2004). La estrategia rusa

I A la desintegración de la URSS, la flota rusa del mar Negro tenía un número de buques de guerra (inclusive submarinos) que variaba, según estiman los especialistas, entre 300 y 635 , mientras que el número del personal asociado a esta flota se estimaba en 47.000-70.000 personas; hasta 1995, la flota rusa había quedado en 48.000, 14 submarinos, 31 navíos de superficie, 43 navíos de patrulla y de costa, 125 aviones de guerra y 85 helicópteros. (Russian Black Sea Fleet).

2 CDI Rusia Weekly, 18 de septiembre de 2003. 
está marcada, de hecho, por dos tendencias contradictorias; por una parte se fomenta el desarrollo de una atmósfera de confianza mediante organizaciones, esquemas de cooperación regional, acciones comunes de preservación de la seguridad contra los llamados "nuevos riesgos" y por otra parte se utiliza la fuerza militar en los conflictos latentes.

A su vez, Ucrania es una avanzada situada entre tres masas geopolíticas: euroatlántica, euro-asiática e islámica. Aunque insignificante como fuerza naval en el Mar Negro (en comparación con Rusia e incluso con Turquía), Ucrania es una pieza importante en el puzzle occidental de los trayectos energéticos. Mediante la puesta en marcha de la terminal petrolifera Odessa, Ucrania tiene la posibilidad de regularizar el flujo petrolifero entre el mar Caspio y el Oriente Medio hacia Europa. A pesar de que, al nivel subregional, Ucrania queda como un jugador con un cierto peso, es evidente que el mismo dependerá, en buena medida, de la trayectoria que seguirá el país según las normas occidentales.

Muy activa, especialmente en las ex repúblicas soviéticas islámicas, Turquía, el más importante pilar americano en la frontera de la masa continental y cultural rusa, representa uno de los líderes regionales en el Mar Negro. Las ambiciones de Turquía tienen como base en buena medida la convicción de que tras la desintegración de la URSS y de Yugoslavia tiene la misión de restablecer la influencia política, económica y sobre todo cultural en los ex territorios del imperio Otomano. Aunque con una presencia significativa en el Mar Negro, la flota turca tiene como principal objetivo garantizar la seguridad de los Estrechos y sobre todo, la capacidad de operar en el marco de las estrategias de la OTAN. Implicada en varios esquemas de colaboración regional (CEMN, BLACKSEAFOR), y consciente del hecho de que ninguna estrategia regional podrá excluir su visión, Turquía tiene una serie de intereses propios que no promueve siempre en consonancia con los aliados riberanos de la OTAN (Rumanía, Bulgaria).

¿Y dónde se posiciona Rumanía en esta constelación geopolítica?

\section{Las dimensiones geopolíticas rumanas en el Mar Negro}

Situada al oeste del Mar Negro y a las bocas del Danubio, Rumanía representa una cabeza de puente para la comunidad transatlántica en el área del Mar Negro. "Isla" de latinidad, rodeada por un mar de eslavos, y de ortodoxismo, Rumanía mantuvo a lo largo de la historia su unidad étnica, lingüística, religiosa y cultural.

Demográficamente, Rumanía está constituida por un "bloque" masivo de rumanos con la misma estructura étnica y el mismo idioma, que conviven con numerosas 
nacionalidades. En el cuadro que presentamos, se pueden observar los datos de la población según etnias, del último censo que tuvo lugar en 2002.

Cuadro 1. La población de Rumanía por etnias, 2002.

\begin{tabular}{|lrc|}
\hline Censo de 2002 & Población & $\%$ \\
\hline Total población & 21.698 .181 & 100 \\
Rumanos & 19.409 .400 & 89,5 \\
Magiares & 1.434 .377 & 6,6 \\
Gitanos & 535.250 & 2,5 \\
Alemanes & 60.088 & 0,3 \\
Ucranianos & 61.091 & 0,3 \\
Serbios & 22.518 & 0,1 \\
Turcos & 32.518 & 0,1 \\
Tártaros & 24.137 & 0,1 \\
Eslovacos & 17.199 & 0,1 \\
Rusos-lipovanos & 36.397 & 0,2 \\
\hline
\end{tabular}

Fuente: Elaboración propia a través del Institutul National de Statistica, Bucuresti, 2003.

Además, más allá de las fronteras rumanas, por los avatares de la historia de este pueblo que tuvo que buscar estrategias de defensa a lo largo de los siglos, viven cerca de 4 millones de rumanos: en Bulgaria (170.000), en Serbia (400.000), en Ucrania - el Norte de Maramures - Transcarpatia-Bucovina del Norte, la región Hertza, Besarabia del Sur -800.000 y la Besarabia rumana -3.000 .000 . A ello añadiríamos otros millones de rumanos que durante los últimos 16 años, tomaron los caminos de la emigración, viviendo en la actualidad como inmigrantes legales e ilegales tanto por el espacio de la UE, como por el de otros países y continentes.

Como elementos económicos característicos mencionamos:

-Importante potencial económico de mercado ( 22 millones habitantes)

-Fuerza laboral relativamente barata y altamente cualificada, con unos ingresos medios mensuales de 150 euros.

-Intensificación de sus relaciones con la UE, mediante intercambios comerciales (más del 70\% del comercio).

-Salida al Mar Negro (los puertos de Constanta, Agigea, Sulina, Mangalia). Facilidades comerciales en relación con los países de la zona de cooperación económica del Mar Negro.

-Potencial turístico importante.

-Recursos naturales significativos (petróleo, gas natural, sal, madera, minerales, carbón). 
-Corredor de tránsito comercial ofrecido por el curso del Danubio y los caminos comerciales tradicionales.

-El vínculo con el Occidente realizado por los canales de Rhin-Main-Danubio y Danubio- Mar Negro, entre el Mar del Norte (puerto Rotterdam) con el Mar Negro (puerto Constanta).

- Conexión de Rumanía con la red internacional de transporte de los recursos de gas natural y petróleo por los oleoductos que unen Irán -Europa Central y Cáucaso - Europa Central. Esta conexión garantizará una seguridad elevada y la independencia energética.

Políticamente, tras la caída del férreo régimen dictatorial de Nicolae Ceausescu, más de una década, el clima político estuvo infectado de suspicacia, intrigas oscuras y visiones conspirativas tenebrosas (Tismaneanu, 1999). Después de una infinita transición, envuelta en corrupción y falta de libertades democráticas, parece ser que, tras la elección del presidente Basescu (2004), representante de la Alianza "Justicia y Verdad", una formación de centro derecha, el país puede vislumbrar la luz al final del túnel.

En la nueva estructura central europea, Rumanía se convirtió en el segundo Estado por superficie, población, potencial económico y militar, tras Polonia. Además, la desaparición de los sistemas dictatoriales ofrece luz verde para la reorientación del eje político interno y externo rumano. Como país europeo, Rumanía tiene, por tanto, la responsabilidad de utilizar su potencial a favor de la democracia y de actuar como un vínculo entre los Estados del Cáucaso y Europa.

Según la teoría geopolítica de Kjellen, Rumanía es un Estado "tapón", posición que define la condición de los Estados pequeños que, desde el punto de vista geopolítico se encuentra en "posiciones intermedias" entre las grandes potencias, lo que reclama un tipo especial de política, la llamada "política-tapón". Este significado lo tienen los Estados situados en el "amplio camino del Danubio y de la llanura húngara" que tuvieron un papel significativo en la defensa contra los avaros, luego contra los magiares, más tarde contra los turcos y los rusos (Kjellen, 1920). A pesar de que Kjellen hacía referencias a una fecha histórica (el año 1916), la característica fijada por él de "bulevar geopolítico" sigue todavía vigente. Dichos Estados tapón se encuentran, pues, en la encrucijada de los grandes intereses geopolíticos. La misma aparición de los Estados balcánicos, Rumanía, Bulgaria y Serbia es la expresión "de la voluntad de las demás potencias de tener un espacio tapón entre Rusia y Constantinopla. De la misma manera se explica la incorporación de Bucovina a Austria. Rumanía tiene esta posición, por excelencia, y este papel de Estado tapón para el cual el historiador rumano Nicolae Iorga utilizaba el sintagma de "Estado de necesidad europea" (Iorga, 1936). Algunos Estados se convierten, pues, en pivotes de configuración geopolítica, to que les confiere oportunidades, pero también riesgos (Badescu y Dungaciu, 2002). 
La dimensión de la proximidad de Rumanía con el Mar Negro varió a lo largo de la historia. Por lo general, se podría afirmar que esta proximidad se situó en relación directa con el estatuto de la república de Moldavia, situada entre el Prut y el Nistru (Dniéster). Hasta 1812, a las provincias rumanas, de Moldavia y Valaquia les perteneció también la parte nórdica del Mar Negro que comprendía las fortalezas Chilia y Cetatea Alba, dos puntos estratégicos extremadamente importantes. A partir de 1812 el territorio estuvo ocupado por Rusia con el acuerdo de Turquía. Evidentemente, se incluía también la franja de litoral correspondiente al sur de la provincia. Como veremos, después de 1918, Besarabia se unió a Rumanía, pero en 1940, cayó de nuevo bajo la dominación soviética. Tras la segunda Guerra Mundial, Stalin la nombró la Moldavia soviética, pero le quitó el sur, es decir, la franja del litoral del Mar Negro y se la entregó a Ucrania. De esta manera, la porción del sur de la República de Moldavia, situada entre el Prut y el Dniéster, pertenece todavía a Ucrania (Marcu, 2005).

El período poscomunista abrió nuevas perspectivas para el Mar Negro. Abierto al comercio internacional, el mar se convierte en una zona de desarrollo regional, importante para Rumanía. Por primera vez en su historia, este mar podría convertirse en un factor generador de unidad, desarrollo y estabilidad en una amplia región de contacto entre dos continentes ${ }^{3}$.

Al juzgar por los documentos oficiales de la administración de Bucarest, la visión rumana parte del hecho de que los riesgos y oportunidades de la zona son similares a los de otros espacios, por ejemplo el Mediterráneo. Esta visión común debería tener entre sus objetivos, mediante la garantía de estabilidad en la región, la seguridad de las rutas energéticas ${ }^{4}$.

El proceso de ampliación de la OTAN y de la UE trajo la comunidad Euro-atlántica a las orillas del Mar Negro, representando el año 2005 una oportunidad especial para un nuevo enfoque del espacio del Mar Negro. Tras 15 años desde los cambios democráticos que afectaron a los países del Este de Europa, con el telón de fondo de las revoluciones de Georgia y Ucrania, se tienen motivos para confiar en un avance considerable de la democratización más allá de estos países.

Además, en el nuevo marco geoestratégico del Mar Negro, existe un claro vínculo entre la comunidad transatlántica por un lado, y los Estados ex soviéticos y los del Oriente Medio por otra. Es ésta una región cuya relevancia política, económica y militar fue sometida a prueba en la lucha contra el terrorismo, en la cual, una serie de Estados, como Rumania, Bulgaria y Ucrania, of recieron una contribución concreta y

\footnotetext{
3 Los habitantes de la antigua Rumanía (Dacia).

4 The Government of Romania: White Paper of Security and National Defence, Bucharest, 2004. p. 5.
} 
valiosa (Basescu, 2005). Rumanía ofreció su espacio aéreo y el acceso a las bases militares junto a la contribución con tropas - más de 2.000 militares, en las operaciones de Afganistán, y de Irak, y las del mantenimiento de la paz en los Balcanes Occidentales.

Más allá de la retórica oficial de Bucarest, está claro que, en este contexto la situación de la región en la inmediata vecindad de la OTAN y de la UE, del Oriente Próximo, la acumulación de problemas sin resolver en Europa (conflictos, tensiones, criminalidad transfronteriza, problemas de déficit democrático), pero también la presencia de unos importantes recursos energéticos de interés para Estados Unidos y los Estados europeos aumentan el valor estratégico de Rumanía en la región del Mar Negro para la comunidad euro-atlántica.

Teniendo en cuenta estos elementos no es una novedad el hecho de que promover la seguridad en su vecindad y consolidar los progresos democráticos representan prioridades estratégicas para Rumanía.

Al haber vivido durante muchas décadas bajo el más cruel régimen dictatorial de la Europa del Este, Rumanía comprende plenamente las dificultades del proceso de reconstrucción económica e institucional. Por ello intenta contribuir al proceso de democratización y consolidación de las instituciones democráticas de los Estados que necesita el apoyo en este sentido.

No obstante, a pesar de todas sus ventajas, Rumanía no posee todavía los medios que le permita una posición propia. Toda la retórica oficial repasa temas ya vistos. La experiencia de su participación en algunos esquemas de cooperación regional, trilateral, demuestra que no se registraron beneficios de ningún tipo: económicos o ideológicos. Desde el punto de vista geopolítico Rumanía no puede tener una posición bien delimitada puesto que su potencial económico y militar ${ }^{5}$ no se lo permite.

\subsection{La actual estrategia rumana en el Mar Negro. La implicación de Estados Unidos}

Tal como señalamos, la región que nos ocupa es una zona de convergencia de los intereses de los grandes actores globales, y las posiciones de algunos jugadores más

\footnotetext{
5 En este contexto, señalamos algunas estimaciones hilares en el artículo "Marea Neagra via Washington" firmado por Radu Tudor y publicado en el diario "Ziua" (El Día) con fecha de 12 de enero de 2005. En este artículo, el autor menciona que "mediante la dotación de las fuerzas navales numanas de las dos fragatas adquiriclas recientemente de Gran Bretaña, junto a la fragata "Marasesti" se crea una agrupación naval "preparadá para asegurar la seguridad en el Mar Negro". Es demasiado aventurado lanzar dichas afirmaciones.
} 
pequeños tienen que configurarse mediante una permanente relación a estos intereses. El hecho de que Rumanía junto a Bulgaria son, en la actualidad, avanzadas de la OTAN en el Mar Negro demuestra claramente que el juego de ajedrez para la dominación del Rimland ${ }^{6}$ está en continuo desarrollo y Rumanía es una pieza importante del mismo.

Desde el punto de vista geográfico e histórico, la región situada entre los mares Báltico y Negro, que incluye el Cáucaso del Sur, no puede excluirse de Europa, siendo cuna de la civilización, además de la barbarie escenificada por la multitud de conflictos a lo largo de la historia. Según analistas políticos, la Europa del Este tuvo y continuará teniendo un papel creativo único a la hora de producir ideas y soluciones experimentales para solucionar los mayores problemas del mundo moderno (Chirot y Jowitt, 1987). Actualmente, la zona está inmersa en una nueva etapa. Su posicionamiento geográfico y estratégico hace de la región del Mar Negro parte imprescindible para la seguridad euro-atlántica. Por ello, Rumanía desarrolla una estrategia común adecuada a la nueva región, renacida en los últimos años. Según analistas políticos rumanos, Rumanía no puede volver a cometer los errores de los últimos diez años (Roncea, 2005). Se trata de dos objetivos fracasados: la implicación en la solución del tema de Transdniester, en la que Rumanía, país extremadamente interesado, con una población mayoritaria en la República Moldavia, se mantuvo fuera del juego, por una parte y el fracaso de su implicación al sur del Danubio, en la antigua Yugoslavia.

La seguridad euro-atlántica está estrechamente vinculada a la seguridad del área del Mar Negro. Muchas de las amenazas transnacionales vienen de esta región. En marzo de 2005, el presidente rumano presentó la alocución "La región del Mar Negro: el avance de la libertad, democracia y estabilidad regional" en el Consejo para las Relaciones Internacionales de Washington. Con este motivo, expuso la importancia que debería tener la existencia de un eje Londres-Washington-Bucarest en la región geopolítica del Mar Negro. Fue entonces cuando la región entró en el centro de la estrategia americana a largo plazo. En su visión, el Mar Negro es una elipse estratégica rodeada de conflictos estratégicos, un cinturón de fuegos latentes que tienen que apagarse, por el interés de la estabilidad regional. A su vez, las teorías rumanas señalaron hace tiempo la importancia estratégica del Mar Negro y el papel fundamental que tiene para Europa, Asia y el Oriente Próximo.

El experto americano Bruce Jackson enunció los seis principios de Estados Unidos para la región del Mar Negro: su apoyo a Rumanía, Bulgaria y a otros países con democracias nuevas, la reforma de las instituciones en la región, la implicación

\footnotetext{
'Rimland - término introducido en el análisis geopolítico por Spykman, para designar la zona del cinturón interior de la representación cartográfica de Mackinder.
} 
directa de Turquía, la prioridad para los conflictos latentes, armonización de los programas de democratización de Estados Unidos y de la UE, y, por último, la concentración sobre el futuro de Ucrania (Jackson, 2005). Al enunciar sus principios, Jackson destacó también la importancia de los vínculos comerciales de la región y el papel del Mar Negro como comienzo del Camino de la Seda. Mientras que la seda y las especias perdieron parte de su valor, las reservas de energía del Asia Central se vuelven cada vez más importantes para los aliados europeos y para la estabilidad del precio mundial del petróleo. Actualmente, los Estados de la UE importan un 50\%, aproximadamente, de sus necesidades energéticas, y hasta 2020 las importaciones llegarán a un $70 \%$. Este aumento podría asegurarse a través de rutas por el Mar Negro. También, Jackson destacó el hecho de que la región se convierte cada vez más en parte de Europa. Junto a estos factores, mencionó la "Revolución de las Rosas" de Georgia y la "Revolución Naranja" de Ucrania, países ribereños del Mar Negro. Por otra parte, desgraciadamente, los más peligrosos conflictos latentes se encuentran en la misma región, desde el este de Moldavia - la franja de Transdniester - Abjazia y Osetia del Sur en Georgia, hasta las cimas montañosas de Nagorno-Karabaj en la frontera entre Armenia y Azerbaiyán. En cada uno de estos conflictos creados por guerras civiles del moribundo Imperio Soviético, tuvieron lugar luchas brutales y limpiezas étnicas que pueden volver a estallar. En Transdniester, Abjazia y Osetia del Sur la criminalidad transnacional encontró refugio y desarrolló bases para el tráfico de armas, drogas y seres humanos. Estas empresas criminales desestabilizan los gobiernos de la región, amenazan Europa a través del tráfico ilícito y a través de la capacidad e intención de vender armas y tecnología.

Para garantizar la seguridad en el Mar Negro, Bruce Jackson propuso una serie de acciones que tuvieran como fin el apoyo hacia las nuevas democracias, impedir las interferencias de las potencias extranjeras y asegurar la apertura de las instituciones euro-atlánticas. En primer lugar se propone la aceleración de las democracias de la región: las perspectivas de democratización en la región del Mar Negro aumentarán sustancialmente con la integración de Rumanía y de Bulgaria en la UE.

Por otra parte, se solicita la reforma y la adaptación de las instituciones (la OTAN y la OSCE) que actúan en la región del Mar Negro, así como la creación de nuevas estructuras regionales para una estrategia en la zona.

La nueva estrategia de Rumanía es "internacionalizar" el Mar Negro con la ayuda de las estructuras euro-atlánticas, y especialmente, de Estados Unidos. Mediante esta estrategia, Rumanía se compromete a defender su posición geoestratégica en las bocas del Danubio y la geopolítica euro-atlántica como país de frontera de estas estructuras, la consolidación de la paz del espacio balcánico, contribuir a la disminución de los conflictos en zonas tan peligrosas como Transdniester. 


\subsection{Las prioridades de Rumanía en el Mar Negro}

Tras los cambios geopolíticos que tuvieron lugar a partir de la década de los 90 , y después de las amenazas terroristas manifestadas a partir de 2001 mediante salvajes ataques que hicieron temblar los cimientos del Occidente, se necesitó un nuevo enfoque global en relación con la defensa y la seguridad.

Según la posición de las fuerzas estratégicas del mundo, ya no puede existir un conflicto global, pero en cambio aparecen y se desarrollan conflictos locales. En las condiciones creadas, Rumanía tuvo que reorientar su propia política exterior y adaptarla a la realidad, teniendo en cuenta, al mismo tiempo, los polos reales de poder del mundo: Estados Unidos, la OTAN, China, la Federación Rusa, la UE, polos que, a pesar de no seguir en disputa entre sí, generan competencia económica, necesidad de pertenencia a un grupo u otro, según sus intereses de seguridad económica y nacional.

Con este telón de fondo, la administración rumana orientó sus prioridades de política exterior en cuatro direcciones. La primera, y la más importante, fue garantizar la prosperidad futura del pueblo rumano. La segunda fue la orientación adecuada de la política exterior rumana. La tercera se dirigió hacia la activación del país en la región, la búsqueda de una política exterior capaz de responder a los intereses nacionales pero, al mismo tiempo, a los intereses de los aliados del país, los europeos, o los miembros de la Alianza Atlántica. La última, pero no por ello menos importante, fue la política del país frente a las realidades del Mar Negro.

La seguridad nacional se sustenta en el partenariado estratégico que Rumanía tiene con Estados Unidos y con Gran Bretaña, que no tiene como objetivo central la evolución económica y la prosperidad, sino la seguridad nacional.

Partiendo de dos realidades, integración en la UE y partenariado estratégico con Gran Bretaña y Estados Unidos, Rumanía desarrolló su propia política regional en el área de los Balcanes Occidentales y el Mar Negro.

"El eje Washington-Londres-Bucarest será una prioridad en la política exterior en los últimos cinco años", declaró el 13 de diciembre de 2004, el día de su victoria como presidente de Rumanía, Traian Basescu. Posteriormente, el presidente rumano reiteró en varias declaraciones públicas su intención de crear esta alianza para el Mar Negro. Su iniciativa dió lugar a un gran debate público. Según el embajador británico en Bucarest, Quinton Mark Quayle, Gran Bretaña centra su interés sobre esta región como consecuencia de los efectos resentidos por el tráfico de drogas y trata de blanca procedentes de la zona. 
¿Por qué Rumanía tiene como prioridad la orientación hacia la región geopolítica del Mar Negro? Los elementos que reclaman la implicación del país en la zona derivan, en primer lugar, del compromiso de tener una contribución positiva a la seguridad europea y a la seguridad de los países de la Alianza del Atlántico del Norte. ¿Qué ocurre de hecho en la región? En primer lugar, señalamos los ya mencionados conflictos latentes y tensiones. Todos se encuentran muy cerca de la frontera este de Rumanía, y por tanto, de la OTAN y de la UE, teniendo impacto sobre la evolución futura del país. Además, la región del Mar Negro está afectada por acciones y actividades que peligran la seguridad de otros Estados europeos. Los tres elementos mayores son: el tráfico de drogas hacia países de la UE, incluso Rumanía; el tráfico de personas, que utiliza el Mar Negro como punto de tránsito hacia los países de la UE, y por último, el tráfico ilegal de armamento, que se desarrolla en volúmenes considerables, sin control, y que alimenta los conflictos locales y regionales, ya sea que hablamos de los Balcanes Occidentales, de Irán, de Irak o sobre cualquier otra zona de conflicto local o regional del mundo. Pero el mar es también un recurso energético esencial para Europa. Desde la zona, se suministra en la actualidad un 50\% de la energía necesaria de la UE y la perspectiva es que en los últimos diez años, un $70 \%$ del necesario de energía de los países de la UE fuera suministrado por esta región (Roncea, 2004). Son éstos los elementos esenciales que, de alguna manera, obligan a Rumanía a formar parte de los procesos políticos que tienen lugar en la región geopolítica del Mar Negro.

Y porque señalamos los procesos políticos, vamos a echar una mirada hacia atrás y recordar la ola de democratización de la Europa del Este del final de los 80. Entonces, Polonia, Rumanía, Checoslovaquia, Bulgaria, todos los países del antiguo Pacto de de Varsovia cambiaron el régimen y pasaron a regímenes democráticos. Tras esa ola de democratización siguió un periodo de instalación de la democracia, después del cual, la influencia que ejercitaron generó una segunda ola de democratización alrededor del Mar Negro, en el espacio ex soviético. Esta segunda ola que comenzó con la "revolución de las rosas" en Georgia, continuó en Ucrania, y se manifiesta en Moldavia, por la reorientación del poder de Chisinau hacia el acceso a la UE. En estas condiciones, Rumanía activa su política exterior en el Mar Negro.

Un punto esencial de la política exterior rumana en el Mar Negro lo constituye el parteneriado estratégico con Estados Unidos. Las etapas de la consolidación de este partenariado tienen dos enfoques. En primer lugar, se trata de una presencia militar legal, activa, de las fuerzas americanas mediante la instalación de bases militares en la zona, y en segundo lugar, en el marco del parteneriado se sitúa el eje ya mencionado Washington-Londres-Bucarest que tiene como fin, la internacionalización del Mar Negro. En la actualidad, el mar está controlado por Rusia, que controla todo lo que ocurre en la zona (a través de las seis bases militares instaladas allí) pero según la administración rumana, la región necesita de la acción de los Estados aliados, de 
su presencia militar, que podrá contribuir a controlar todo tipo de tráficos ilegales. Aquí, existe de alguna manera también la acción de Turquía, que controla los Estrechos de Bósforo y Dardanelos.

El 23 de octubre de 2005, se estableció el emplazamiento de las bases militares americanas en la región rumana de Dobrogea, en la proximidad del Mar Negro: Babadag, Constanta y Fetesti. Según comentarios de Deutsche Welle ${ }^{7}$ el interés de Estados Unidos en propiciar la seguridad y la prosperidad en el Mar Negro está vinculado al transporte del petróleo. El puesto de radio alemán precisó, citando a Washington File, que el volumen de los transportes desde y hacia el Mar Negro aumentó más de dos veces desde 2001, en condiciones en que a través del Estrecho de Bósforo pasan a diario 150 buques, de los que 25 son petroleros. Un eventual ataque terrorista contra las facilidades navales o portuarias amenazaría directamente los intereses y la seguridad económica de Estados Unidos y de todos los países que utilizan los recursos energéticos de esta región. Las bases entraron en función en diciembre de 2005 y, finalmente se emplazaron en varios puntos de Rumanía, además de las localidades del mar Negro, ya mencionadas, también en Smirdan, en la proximidad de la ciudad de Galati (por el curso del Danubio), como en los Cárpatos.

\section{Tensiones y conflictos en la frontera del Mar Negro y sus implicaciones para Rumanía}

Hemos señalado a lo largo de este artículo, la existencia de conflictos y tensiones en la región geopolítica del Mar Negro. Durante la Guerra Fría, las grandes potencias proyectaban sus tensiones a escala global. Tras el colapso de la URSS y el fin de la Guerra Fría se puso en evidencia la falta de razones ideológicas profundas en el origen de aquellos conflictos, los cuales, en lugar de haber desaparecido, se han transformado y vuelto más complejos, evidenciando problemas económicos, étnicos, religiosos o nacionales.

Las tensiones de la región, de naturaleza étnico-cultural, están estrechamente vinculadas con las revueltas producidas ante el nuevo orden político y la incertidumbre

\footnotetext{
7 Deutsche Welle. 11.01 2002. El Pacto Ribbentrop Molotov (entre Alemania y la URSS) El pacto fue firmado en Moscú, en 1938 por Ribbentrop y Molotov en presencia de Stalin. Por el pacto de no agresión, ambos países acordaron no atacarse, ni independientemente ni en alianza con otros estados; no apoyar a otro tercer país que pudiera atacar a la otra parte del pacto; continuar las consultas sobre los temas de interés común; no unirse a ningún grupo de potencias que directa o indirectamente pudiera amenazar a cualquiera de las partes firmantes; y a resolver sus diferencias mediante la negociación. El pacto tendría una duración de 10 años. A este tratado se le unió un protocolo secreto que dividía la Europa oriental en zonas de influencia germana y soviética. Se acordó la partición de Polonia y se dejó a Finlandia, Estonia, Letonia, Lituania y la Besarabia en el área de influencia soviética.
} 
de los acontecimientos y su dudosa evolución. Tanto el espacio de la antigua URSS, particularmente el área caucásica, como algunos países periféricos (Moldavia) constituyen el área de localización de este tipo de "desencuentros" (Plaza, 2000).

Además, existen tensiones entre las llamadas potencias de la región. Si tradicionalmente tuvo lugar una competición frontal entre tres imperios rivales, Otomano, Austro-Húngaro y Ruso, actualmente, participan dos potencias que forman parte de la región: Rusia y Turquía.

Aunque los dos países buscan, al menos, una esfera de influencia, en el caso de Rusia las ambiciones de Moscú tienen un alcance mucho mayor debido a los recuerdos imperiales, a la presencia en la región de varios millones de rusos y al deseo de reinstalarse como una potencia global. En cambio, si bien las aspiraciones turcas de ejercer una influencia al nivel regional mantienen algunos vestigios imperiales mucho más antiguos (el apogeo del Imperio Otomano fue en 1590), tienden a estar enraizadas en un sentimiento étnico-lingüístico de identidad con los pueblos turcos de la región. Por tanto, las tensiones creadas y los intereses en juego tienen que ver con el poder político, con el acceso a unas riquezas potencialmente importantes y con la seguridad.

Las tensiones de la región afectan a Rumanía, en relación con Ucrania, sobre todo. Existe, asimismo, la cuestión de Besarabia y de Transdniester, las dos regiones de la República Moldavia, de identidad rumana.

\subsection{Las tensiones Ucrania - Rumanía y la teoría de Mackinder}

Para comprender el contexto actual de las tensiones entre estos dos países de la región del Mar Negro, es necesario explicar brevemente la teoría geopolítica del geógrafo inglés, Halford Mackinder. Esta teoría que influyó, desde 1904, el año de su primera formulación, en tres generaciones de estrategas militares, se conoce en la actualidad, como la "teoría del heartland". Según Mackinder, las partes que se encuentran en conflicto son determinadas geográficamente. El poder marítimo se encuentra en conflicto con el "Estado-pivote", el Estado que controla la "Isla-mundo". La masa territorial euro-asiática y africana forma el núcleo de este World-Island. El control sobre este territorio permitirá el empleo de algunos recursos humanos y materiales inmensos de un solo Estado en detrimento de todos los demás. Siendo accesible a los ataques venidos desde el mar y consiguiendo llegar a los mares libres, "el estado pivote" conllevará a la destrucción de la balanza del poder en su favor, resultando de aquí una expansión sobre las tierras euro-asiáticas marginales, el empleo de sus recursos inmensos en la construcción de una flota propia y la aparición en el horizonte del imperio mundial. Partiendo de una aplicación de su teoría a las relaciones con- 
flictivas ruso-británicas de antes de la primera guerra mundial, Mackinder añadirá su teoría del "estado-pivote" del 1904 a la del heartland de 1918, cuando formula su teoría: "Quien controla la Europa del Este controla el Heartland. Quien controla el Heartland controla la Isla-Mundo. Quien controla la Isla-Mundo controla el Mundo" (Mackinder, 1919).

En términos pragmáticos se trata de una lucha por el control de la Europa del Este entre las potencias territoriales. A finales de los 80, Zbgniew Brzezinski retomaba la teoría de Mackinder, afirmando: quien controla Eurasia, controla el mundo. Si la Unión Soviética domina las periferias de este territorio ganará no sólo el control sobre unos recursos humanos, económicos y militares inmensos, sino también el acceso a los puntos geoestratégicos del hemisferio occidental (Brezezinski, 1989). El Imperio Ruso queda como uno de los actores constantes de las teorias de Mackinder. Las tres dimensiones de expansión histórica son las mismas: hacia el Occidente (Polonia, Silesia, Moravia, Rumanía, Croacia, Hungría y Serbia), hacia el sur-oeste (las regiones del Mar Caspio e Hindicus) y hacia China.

Tras el desplome de la URSS, una buena parte de la diplomacia americana parece volverse hacia una visión de tipo Mackinder sobre el nuevo Imperio del Heartland. El conflicto renacido entre las fuerzas marítimas y las territoriales conoce momentos muy difíciles en Georgia, Moldavia y otras zonas de la periferia imperial de donde tiende a extenderse ahora hacia la zona danubiana.

El ingreso de Rumanía en la OTAN y su integración en la UE, en enero de 2007, crearon para algunos de los herederos de la antigua Unión Soviética, como Ucrania, tensiones vinculadas a la Isla de las Serpientes y al Delta del Danubio.

\section{2. La Isla de las Serpientes y el canal Bastroe. Piedras de toque de la diplomacia rumana}

La Isla de las Serpientes está situada en el Mar Negro, a una distancia de 40 kilómetros (22 millas marinas) del litoral rumano del Mar Negro. En la Edad Media, la soberanía de la isla perteneció sucesivamente a los principados rumanos de Valaquia y Moldavia. El periodo moderno de la historia rumana trajo modificaciones no siempre claras sobre soberanía de la isla. De esa manera, mediante el Tratado de Adrianópolis (1829) Rusia extendió su frontera danubiana desde el brazo de Chilia hasta el brazo San Jorge, y aunque no se hiciera referencia a la Isla de las Serpientes, está claro que la misma se incluyó bajo el dominio ruso. Los rusos construyeron en 1842 un faro allí para dirigir la navegación en el Mar Negro. El Tratado de San Esteban, consecuencia de la guerra ruso-rumano-turca, tratado que no fue reconocido 
por las grandes potencias europeas, hacía constar la cesión de Turquía a Rusia, de la Isla de las Serpientes, junto al Delta del Danubio y la región de Dobrogea. A su vez, Rusia impuso y ofreció a Rumanía el Delta, la región de Dobrogea y la Isla a cambio de los departamentos del sur de Besarabia (la actual Moldavia) que le había cedido en 1856. El Tratado de paz de Berlín, firmado el 13 de julio de 1878 reconoció lo que se estableció en San Esteban. Entre 1878 y 1948 no existía ningún tipo de referencia en los documentos diplomáticos rumano-rusos o soviéticos, en cuanto a la Isla de las Serpientes, que, por otra parte, ya pertenecía a Rumanía. En 1940, Rumanía fue obligada a ceder a la Unión Soviética, el territorio comprendido entre Prut y Dniéster, pero de nuevo, no se hizo mención a la Isla. Por el Tratado de Paz de Paris de 1947, se decidió que la "frontera soviético-rumana se fija de conformidad con el acuerdo soviético-rumano de 1940" es decir, de nuevo, sin referencias a la Isla, sin ninguna mención concerniente a una eventual soberanía soviética sobre la Isla, que había sido ocupada de facto por las tropas soviéticas en agosto de 1944. De esta manera, en 1948 se firmó en Moscú el Protocolo entre Rumanía y la Unión Soviética, referido a la línea de frontera entre la URSS. En el Protocolo, se estipulaba que "La Isla de las Serpientes, situada en el Mar Negro, al este del Danubio, entraba bajo el dominio de la URSS" (artículo 1, línea b). Este protocolo no fue nunca ratificado por Rumanía. En los años del régimen comunista que siguieron, no se trató el problema de la ilegalidad de la soberanía de la Isla. Con el telón de fondo del paulatino distanciamiento del régimen de Ceausescu de los demás miembros del bloque socialista, mediante el problema del reparto plató continental de Rumanía en el Mar Negro, se abordó la cuestión de la Isla de las Serpientes.

Por lo tanto, el periodo del comunismo en la región consignó la creciente importancia estratégico-militar de la Isla. Mientras, allí se creó y desarrolló una fuerte base militar, de control aéreo y naval. Las capacidades militares de investigación aérea de Ucrania permiten en la actualidad el control del perímetro que comprende el Mar Negro y el Mediterráneo, hasta las costas de Libia. En la Isla se emplazaron estaciones de interferencias y escuchas de las transmisiones militares, pero también civiles, que cubren un área extremadamente mayor. Las instalaciones militares propiamente dichas están protegidas por importantes unidades navales, ucranianas, entre al que nombramos dos submarinos, una fragata y buques patrulla. Las comunicaciones aéreas se aseguran mediante un helicóptero (Padureanu, 2005).

Tras la caída del régimen soviético, Ucrania se convirtió en uno de los más grandes actores del escenario europeo post-soviético, pasando a ser vecino directo de Rumanía y retomando los antiguos problemas de las relaciones rumano-soviéticas. La Isla de las Serpientes es uno de estos problemas. Tenemos que señalar el hecho de que Rumanía no tiene pretensiones territoriales con Ucrania. Como país firmante de los Acuerdos de Helsinki, Rumanía respeta el estatus quo obtenido tras de la segunda Guerra Mundial. El litigio, o el diferendo sobre la isla, no se refiere a su retrocesión 
a Rumanía sino al reparto de los espacios marítimos entre los dos países. El Tratado entre Rumanía y Ucrania, firmado en 1997 consagra la realidad territorial existente en el momento del derrumbe de la URSS, inclusive la pertenencia jurídica de la isla de las Serpientes al último entre los firmantes.

Para poder tomarse en cuenta la delimitación del plató continental y de las zonas económicas exclusivas de Ucrania en el Mar Negro, la Isla de las Serpientes debería ser habitada y debería tener todos los recursos necesarios para la existencia de la vida. No obstante, en la isla, tal como señalamos más arriba, sólo hay vegetación de estepa, no hay árboles y tampoco agua. Los alimentos que necesitan los soldados destinados allí, llegan cada semana en un helicóptero, pero cuando llueve y hay tormentas, aparecen las dificultades. Según fuentes diplomáticas rumanas, Ucrania intenta demostrar la posibilidad del desarrollo de una vida social y económica en la isla. De esta manera, trajo tierra en la isla, creó un ponto artificial y se plantaron árbó les. El Ministro ucraniano de exteriores, Konstantin Griscenko afirmó que en la Isla se encontraron recursos de agua potable, y la población está en aumento.

En febrero de 2004, ni Rumanía ni Ucrania renunciaban a la Isla de las Serpientes. Ucrania quiso modifiear el régimen de la Isla de las Serpientes para que la misma fuera considerada un territorio habitable y no una roca inhabitada. Rumanía, a su vez, no aceptó el cambio jurídico de la Isla, que tendría consecuencias en el plano internacional. Desde el punto de vista del país, la Isla de las Serpientes representa el elemento central en los esfuerzos de solucionar la delimitación de la parte continental del Mar Negro.

Finalmente, en agosto de 2005, Rumanía tomó la decisión de apelar a la Tribunal Internacional de la Haya para reglamentar la disputa con Ucrania en cuanto a la delimitación de las zonas económicas del Mar Negro. Tras 24 rondas de negociación desarrolladas a lo largo de los últimos seis años, Ucrania manifestó una posición inflexible, pretendiendo una superficie de hasta $200 \%$ mayor que la pretendida en su día por la URSS.

La disputa rumano-ucraniana por la delimitación del plató continental del Mar Negro se mueve alrededor de los recursos de petróleo y gas natural existentes en la región. Para algunos perímetros de la zona con recursos, evaluada en 13.000 kilómetros cuadrados, la empresa inglesa Palladin Resources demostró su interés, y de hecho ganó, en una primera fase, dos perímetros, Gasca y Doina. Posteriormente, Palladin Resources, se retiró, debido a su incertidumbre en relación con la pertenencia de los recursos. Por otra parte, desde 1980, la empresa rumana Petrom explota un recurso llamado Lebada (Cisne). El mismo suministra aproximadamente 500.000 toneladas de petróleo anuales, lo que representa un $10 \%$ de la producción nacional anual de petróleo. Petrom descubrió otro recurso, llamado Gaviota, estimando los expertos una 
posibilidad de extracción de 250.000 de toneladas al anuario. A su vez, los ucranianos implantaron una sonda en un antiguo perímetro rumano, llamado Muridava (en rumano) y Olimpskia en ucraniano.

El gobierno rumano concedió en 1998, una licencia de explotación y exploración del plató continental del Mar Negro a la empresa Total Fina Elf, un gigante en la industria petrolera. Mediante contrato, el estado rumano ofrecerá el derecho de explotación de los recursos por esa empresa, mientras que la empresa sostiene un lobby fuerte de tal manera que Rumanía pueda volver a tener la isla. El derecho de explotación se deberá asegurar al gigante petrolero por un periodo de 49 años, a cambio de petróleo. De esa manera Rumanía podría asegurarse un $25 \%$ del necesario interno de petróleo. De momento, sigue el contencioso sobre esta pequeña isla con gran importancia estratégica.

Además de la Isla de las Serpientes, los problemas de frontera con Ucrania comprenden también el Delta del Danubio. De esta manera, las oficialidades de Kiev planificaron la construcción de un canal que vincule el Danubio al Mar Negro mediante el canal Bastroe -que atraviesa una reserva natural- para permitir a Ucrania tener una vía de acceso navegable al mar. Este canal tendrá 10 kilómetros y Ucrania intenciona acabarlo hasta 2007. Actualmente, los buques de Ucrania tienen que pasar por Rumanía para llegar al Mar Negro. Por otra parte, el buque ucraniano Rostok, varado en septiembre de 1991, bloquea la navegación en el brazo Sulina.

En mayo de 2004, Ucrania puso en práctica su proyecto de "conquista" de las bocas del Danubio. De esta manera, se empezó a construir un canal navegable directo, entre el Danubio y el Mar Negro, situado en el brazo Bastroe. El proyecto resulta ser muy criticado tanto por la parte rumana, como por la comunidad internacional. La realización de este canal permitiría a Ucrania el acceso directo del Danubio al Mar Negro sin que tuviera que pasar por el canal navegable rumano Sulina, donde los buques ucranianos pagan tasas de tránsito. Como señalamos más arriba, los buques ucranianos tienen que pasar por Rumanía para poder llegar al Mar Negro, después del abandono, en el año 1997, del canal ucraniano Prorva, convertido en impracticable después de una utilización de 40 años. Por lo demás, el argumento principal de la parte ucraniana para la construcción de este canal es el económico, sosteniendo Kiev que mediante el tránsito del canal rumano Sulina, Ucrania registró perdidas de 600 millones dólares en los últimos 5 años.

Pero más allá del aspecto económico, la construcción de este canal navegable tiene una importancia estratégica especial en la región, puesto que consolida la posición estratégica de Ucrania en el Mar Negro.

El proyecto del canal navegable ucraniano recibió durante los últimos años vivas protestas en el marco de la comunidad internacional, debido al impacto devastador 
que tendrá esta construcción sobre el medio ambiente. La construcción del canal supone la instalación de un verdadero astillero que pasaría por la mitad del Delta del Danubio y afectaría gravemente el ecosistema. El canal será utilizado por 1000 embarcaciones anuales, y las ganancias de Ucrania se elevarían a 50 millones dólares anuales.

Importantes organizaciones internacionales para la protección del medio ambiente, entre los cuales UNESCO, el Fondo Mundial para la Naturaleza y Bird Life International se opusieron a este proyecto, poniendo de manifiesto que el mismo representa una amenaza para el ecosistema del Delta del Danubio. Inscrita desde el año 1991 en el patrimonio mundial UNESCO, la reserva natural del delta, con una superficie total de 626.403 hectáreas, repartida entre Rumanía y Ucrania, comprende aproximadamente, tal como se señaló 90 especies de peces y más de 300 especies de pájaros, entre los cuales algunas son ejemplares amenazados por la desaparición. Con su flora y fauna lacustre, teniendo las marismas más extensas de Europa, esta reserva representa el más importante sistema de purificación de aguas del continente europeo, según afirma UNESCO. La supervivencia de las especies en vías de desaparición, por ejemplo el cormorán enano, el águila marina o varias especies de pelícano, será más difícil aún tras la construcción del canal Bastroe, según expertos en la protección del medio ambiente.

Las autoridades rumanas sostuvieron que Ucrania no debería comenzar las obras del canal hasta que no existiera un acuerdo de las organizaciones internacionales y un estudio de viabilidad que garantizara que esta construcción no afectaría el ecosistema del delta del Danubio.

Cuando, en 1997 se firmó el Tratado político Rumanía-Ucrania, las negociaciones fueron dominadas por la posición inflexible de Ucrania, en cuanto a problemas de frontera y tratamiento de las minorías étnicas. Aunque la frontera común rumanoucraniana representa el resultado impuesto por el Pacto Ribbentrop -Molotov fue Ucrania la que impuso el texto del tratado ya mencionado, que Rumanía considera "humillante".

\subsection{La implicación de Rumanía en la región de Transdniester}

La desmembración de Rusia, la retirada estratégica de las tropas soviéticas de la Europa central y Oriental, el estallido de la ola de revoluciones de 1989, el proceso de redefinición de las relaciones internacionales, la desaparición de la bipolaridad y la aparición de nuevos centros de poder creó un verdadero caos geopolítico que todavía tiene implicaciones directas sobre la seguridad europea y mundial. En el 
plano regional, Rusia consiguió la creación y el mantenimiento de algunos enclaves en la línea fundamental de demarcación geopolítica del istmo ponto-báltico, en el Kaliningrado y en el sur, en el territorio de la República Moldavia, en Transdniester.

El mayor objetivo de Rusia fue retener a la República Moldavia bajo la tutela política del antiguo centro de decisión post-soviético, utilizando la región de Transdniester, como una mecha que puede estallar en cualquier momento. Tiraspol, la "capital" de la región separatista, se convirtió en la clave de la región y del sistema de dominación rusa. Lós líderes secesionistas constituyeron con el apoyo militar ruso un ejército dotado con técnica militar moderna, formada por tanques, instalaciones de raquetas, tropas de genio e incluso aviación de guerra, utilizando el potencial industrial de las empresas de la región para aumentar su arsenal, ya de por sí considerable.

El problema de Transdniester es un problema fundamental de la seguridad de Rusia. El río (Nistru) Dniester constituye la línea estratégica que separa los dos grandes espacios geopolíticos, el espacio eslavo del resto del mundo europeo. El control del Dniester se convirtió en objetivo prioritario de todos los imperios que se sucedieron en el perímetro del istmo ponto - báltico. Tras la independencia de Ucrania, la perdida de Odessa y de los importantes puertos militares en el Mar Negro, de Azerbaiyán, ya bajo influencia turca, y de Georgia, donde se instalarán bases americanas, para Rusia mantener el control de Tiraspol como cabeza de puente hacia Europa Sur-Oriental conlleva una significación estratégica importante.

El conflicto militar de la franja del este de Moldavia -Transdniester- comenzó en junio de 1992. El rechazo del presidente Voronin de firmar el llamado "plan Kozak" (2003) realizado con vistas a solucionar el conflicto transnistrio, representó el comienzo de una nueva orientación exterior de Moldavia, una pro-occidental. El memorando preveía ciertas condiciones en las que la región separatista se hubiera podido independizar de Moldavia para proclamar su independencia o para afiliarse a otro estado. Actualmente, a pesar de la multitud de planes, no hay una estrategia clara para la región, hecho que entorpece la potencial integración de Moldavia a la UE, a medio y largo plazo.

La agravación del conflicto, la corrupción y la pobreza, la precaria seguridad de las fronteras llevaron a establecer un clima favorable para desarrollar actividades de crimen organizado en Moldavia, especialmente del tráfico de armamento y munición, migración ilegal y tráfico de seres humanos (Menar, 2005).

Rusia, en una incansable búsqueda de su espacio geopolítico vital, cruzó el espacio de Rumanía en numerosas ocasiones a lo largo de la historia, dejando atrás heri- 
das que todavía siguen abiertas. El régimen comunista impuesto con fuerza al Ejército ruso y los rumanos deportados en campos de concentración hurgaron aún más en las heridas. La historiografía sostiene que Rumanía entró en guerra contra la URSS junto a Alemania Nazi en junio de 1941, pero la invasión de Rumanía por las tropas soviéticas se produjo en junio de 1940, y debe considerarse, igualmente, como un acto de guerra. Rumanía tuvo que ceder a la fuerza Besarabia y Bucovina del Norte, (Chisinau, Cernauti y Cetatea Alba).

Las agresiones soviéticas fueron posibles tras la firma del Pacto de no agresión entre Hitler y Stalin firmado por sus ministros de Exteriores Joaquín von Ribbentrop y Viaceslav Molotov. Los soviéticos reconocieron el Pacto Ribbentrop - Molotov, apenas en 1990. De esta manera, el protocolo secreto fijaba sus zonas de influencia de esta manera: además de los países bálticos y Finlandia de Norte, la Unión Soviética manifestaba su interés por Besarabia, ya que la parte alemana declaró su total desinterés sobre la zona. El reparto realizado entre Stalin y Hitler hizo estallar la guerra mundial. Como bien se conoce, Polonia, el más grande país de la zona, fue invadida la primera, fijando de esta manera la fecha del estallido de la conflagración mundial, pero de la misma categoría forman parte los países bálticos y Rumanía, Estados invadidos por la Unión Soviética. Como consecuencia, y aconsejada por Alemania que aceptara las condiciones impuestas por la URSS, Rumanía aceptará el 28 de junio de 1940 la evacuación de los territorios de Besarabia y Bucovina del Norte a la URSS. Los políticos de Bucarest esperaban que, al evitar la utilización de la palabra "ceder" anularían las pretensiones posteriores de Moscú. Pero no fue así, y Rumanía perdió 50.762 Kilómetros cuadrados (Besarabia $-44.500 \mathrm{Km}$. cuadrados, el Norte de Bucovina $-6.262 \mathrm{Km}$. cuadrados, con más de 4.000 hectáreas de terreno agrícola, un 20,5\% de la superficie agrícola del país), 3.776 .309 habitantes, entre los cuales un 53,49\% de rumanos, un $10,34 \%$ rusos, un $15,30 \%$ ucranianos y rutenios; un $7,27 \%$ judíos, un $4,91 \%$ búlgaros; un 3,31\% alemanes, un 5,12\% otras etnias.

Durante las operaciones militares del Ejército Rojo de junio de 1940, los habitantes de las regiones de Besarabia y Bucovina del Norte sufrieron un terrible calvario. Además de las muertes de niños y de adultos, se practicó la deportación masiva de cientos y miles de habitantes de la zona, (3.470 familias, con 22.648 personas) al considerarlos peligrosos para el proceso de instauración del régimen comunista. Entre 1944 y 1951, en el territorio de la ya creada (por el aparato estalinista) República Soviética Socialista Moldavia se realizaron otras 3 series de deportaciones de moldavos hacia Siberia, Kazajstán, para que, en su lugar se trajesen rusos y ucranianos. Se persiguió desarraigar a los rumanos de Besarabia de sus tierras natales y modificar la composición étnica de Besarabia a favor de la URSS (Damian, 2005). 
Actualmente, sin sus territorios, que mediante los acuerdos firmados a comienzos de la década de los 90 entre Gorbachov e Iliescu ${ }^{8}$, Rumanía se considera un actor importante en cuanto al conflicto de Transdniester. Pero, por falta de conocimiento histórico, y por los intereses y deseos de poder de Rusia y de Ucrania, Rumanía estuvo, por ahora, claramente excluida de cualquier tipo de negociaciones. Y hay que recordar que, aunque sin éxito, el país, pilar de estabilidad en la región del Mar Negro, como miembro de la Alianza Atlántica intentó participar varias veces a la mesa de tratativas de la solución del conflicto de Transdniester. Se espera que, con el ingreso efectivo del país en la UE, como país miembro, pueda participar a dichas negociaciones. El interés de Rumanía es que la República Moldavia vuelva a tener la soberanía sobre todo su territorio.

Desde Transdniester, se asegura que no cabe ninguna posibilidad de integración de la región en Moldavia. Es más, Grigori Marakuta, presidente del Soviet Supremo de Tiraspol, señaló, que la única vía de salida del conflicto es el reconocimiento internacional de la región, como estado independiente (Marakuta, 2005).

En abril de 2006, Rumanía, teniendo en cuenta las recomendaciones de una serie de organizaciones internacionales (ONU, OSCE, UE,), además de los Estados Unidos, intentó llevar a cabo un plan de solucionar el conflicto de Transdniester, junto a Moldavia y Ucrania, bajo el titulo "Partenariado regional para una solución mutua" (Severin, 2006). Aunque es pronto para saber el contenido del plan, se supone que su fin se refiere a la erradicación de la criminalidad y a la retirada de las tropas rusas que permanecen en el país, siendo estos, pasos importantes para la instauración de la democracia en la región.

Hay que señalar el hecho de que Rumanía, además de su conciencia europea, atiende, asimismo, los intereses nacionales, sobre todo en relación con los rumanos de las provincias de Besarabia, Bucovina, y la región de Herta, raptadas por la URSS y divididas actualmente entre Ucrania y la actual república de Moldavia. Por ello, teniendo en cuenta la identidad de lengua, cultura y tradiciones se considera que el futuro europeo de la República Moldavia es una deuda moral para Rumanía. No hay que olvidar el genocidio cultural llevado a cabo en Besarabia y en Transdniester, con el cierre de escuelas, colegios e institutos rumanos (ya a la altura del verano de 2004) en los planes de rusificación de Moldavia. En Transdniester existe, asimismo, una censura de la prensa escrita en rumano con grafía latina. En las bibliotecas de Transdniester, territorio rumano, se encuentran escritos en el idioma rumanos, sólo las obras de Lenin y Brejnev (Iovcev, 2005)

\footnotetext{
8 En abril de 1991, se firmó el Acuerdo entre la URSS y Rumanía, entre los por aquel entonces presidentes Mijail Gorbachov e Ion Iliescu mediante el cual, Besarabia y la Isla de las Serpientes quedarian en la compasión de la URSS.
} 
Los hechos y la historia reciente demuestran que Rumanía no es, por lo menos por ahora, un líder regional o un actor decisivo en el Mar Negro, pero sus últimas estrategias, desde su ingreso en la OTAN, y como futura frontera de la UE en el este, tiene muchas oportunidades de convertirse, a través de proyectos inteligentes, en un actor en la zona. Por ello, cuantas más iniciativas tenga en la región, más posibilidades tiene de implicarse en Transdniester, pues no existe ninguna justificación para mantener alejada a Rumanía, país directamente implicado, fuera de las negociaciones.

\section{La gravitación de Rumanía en el sistema geopolítico del Mar Negro, en el siglo XXI. Consideraciones finales}

El más antiguo sistema geopolítico de Europa, con una importancia estratégica para Rumanía, ya se ha convertido en una realidad para la comunidad euro - atlántica.

Si tal como señalamos, en el siglo XIX representó el teatro de confrontación de las grandes potencias, puesto que las "llaves" del sistema están representadas por los Estrechos de Bósforo y Dardanelos (controlados por Turquía y objeto de disputa con Rusia) y la península Crimea (con las bases navales de Sebastopol, Simferopol y Balaklava) - objeto de disputa entre Rusia y Ucrania, en la actualidad, el Mar Negro se convirtió en el polo de atracción de las alianzas militares y de los intercambios económicos, cobrando una nueva perspectiva para el siglo XXI.

Actualmente, los intereses de Turquía y de Rusia se sobreponen menos en la cuenca del Mar Negro y más en la zona de las repúblicas turco-musulmanas del Asia Central, siendo los medios de influencia de Turquía en la zona netamente superiores frente a los de Rusia contra Turquía.

Invocando la seguridad de la navegación y la protección del medio marino, Turquía solicita que las exportaciones petrolíferas de la CEI se realicen sólo a través de los estrechos. Como consecuencia, Ankara propuso la creación de un oleoducto que uniera los campos petrolíferos del Cáucaso con un puerto mediterráneo, es decir, turco. Este proyecto se llevó a cabo en 2005 mediante la puesta en marcha del oleoducto Bakú-Tbilisi-Ceyján que esquiva a Rusia y ofrece un importante papel estratégico a Turquía.

La libre navegación en los estrechos no se podrá llevar a cabo si la salida hacia el mar Mediterráneo, respectivamente por el Mar Egeo, no conocerá una cierta libertad. Este elemento es esencial en el diferendo griego-turco en cuanto a la delimitación de 
las zonas de soberanía marítima, así como en las reivindicaciones de Ankara frente a algunas islas griegas situadas en la proximidad del litoral turco. Si el Mar Negro pudo ser considerado un campo de batalla en las grandes confrontaciones entre el Este y el Oeste, se convertirá en el espacio de las colaboraciones locales, regionales e internacionales.

Aunque, en la actualidad se manifiestan conflictos locales, como son los de la parte oriental de la zona entre armenios y azeros, abhazos y osetios, sin hablar del de los kurdos en Anatolia, esta región, un verdadero mosaico étnico-religioso, que, a lo largo de la historia, se encontró en la encrucijada de imperios, conoció a la vez con la desaparición del último, la libertad para todas las naciones. Ni la Federación Rusa, ni Turquía, ni Irán pueden permanecer insensibles. Esta región es, no obstante, una zona de vínculo entre el Este y el Occidente, desde el Mar Negro hasta Asia Central, más allá del Golfo Pérsico. Al mismo tiempo, el Mar Negro tiene recursos de hidrocarburos. Los países situados al Este del mar siguen todavía bajo las incertidumbres económicas y políticas, como consecuencia de la guerra de Yugoslavia y, por otra parte, de las modificaciones territoriales regionales que tuvieron lugar tras el desplome de la URSS.

En este contexto geopolítico perturbador está claro que la dominación en el Mar Negro tiene una gran importancia estratégica, y Rumanía, pilar de estabilidad en la OTAN y frontera de la UE tiene varios papeles que desempeñar.

Por tanto Rumanía representa la puerta comercial sur - oriental, a través de:

- La constitución de las zonas libres económicas de Sulina al Mar Negro, Ungheni y Giurgiulesti en Galati, las realizadas con Hungría, las de Silistea - Calarasi con Bulgaria, y la realizada con Yugoslavia.

- La conexión de Rumanía a la red internacional de transporte de los recursos de gas natural y de petróleo a través de las magistrales Irán - Europa Central y Cáucaso - Europa Central, garantizará una alta seguridad e independencia energética (mediante la central nuclear de Cernavoda).

- Los intercambios comerciales se realizan, en principio, a lo largo del litoral occidental y del norte: Burgas y Varna en Bulgaria, Constanta en Rumanía, Odessa y Crimea e Ucrania y Novosibirsk (complejo petrolífero accesible a los buques de 200.000 toneladas) en Rusia.

El tráfico comercial en el Estrecho de Bósforo tiene un valor próximo al de los Estrechos de Panamá y Suez, mientras que el desarrollo de las exportaciones de productos petrolíferos del la Comunidad de Estados Independientes (CEI) reactivan el puerto de Constanta, el mayor puerto en el Mar Negro y, posiblemente, la reconstitución de la flota rumana. 
Para Rumanía, el desarrollo de la relación entre los mares Caspio-NegroMediterráneo es vital desde el punto de vista económico y comercial. Esta perspectiva puede ser vital en las relaciones de Rumanía con el mundo occidental, por el desarrollo de la navegación marítima y fluvial.

Según los especialistas político-militares franceses, Rusia no abandonó nunca su proyecto de "abrir una ventana" hacia el sur (Delorme, 1996). Las rivalidades actuales ruso-ucranianas sobre el futuro de la Flota del Mar Negro y de sus bases militares tienen como objetivo la autoridad sobre este mar. Ucrania desea obtener la dominación sobre los recursos marítimos, mientras que Rusia no desea perder su único modo de acceso hacia los mares cálidos.

En la actualidad, Rusia está rodeada por proyectos occidentales. Molesta por el proyecto político-militar de conjunto, de internacionalización del Mar Negro, Rusia criticó firmemente el avance de la fuerzas de la OTAN hacia las fronteras de la ex URSS, la actual CEI. Rumanía es una parte del complejo plan que supera las estrategias regionales previstas por Rusia, que mira irritada, desde sus alturas imperiales, los despliegues de tropas en el antiguo satélite del bloque soviético.

El Mar Negro se ha convertido en un nuevo punto importante del mapa de las estrategias de defensa y de seguridad de las estructuras especializadas europeas y americanas. Sin lugar a dudas, ampliando este marco, los analistas geopolíticos observan con cierta facilidad el vínculo entre las así llamadas revoluciones pacíficas de colores en el Cáucaso y en Ucrania y los intereses suscitados por los recursos minerales ricos del Cáucaso y del mar Caspio. El avance de la OTAN y de EEUU hacia las fronteras de la ex URSS parece formar parte de la nueva estrategia.

Y en este juego, la edificación del papel de Rumanía y de su aliado, el Mar Negro, cambiará, sin duda la arquitectura de seguridad de toda la región.

\section{Bibliografía}

Asmus, D. R. y Jackson, P. (2004) The Black Sea and the frontiers of Fredom. Towards a new Euro-Atlantic Strategy Policy Review, 25 june-july 2004. Washington D.C.Hoover Institution.

Asmus, D. R. Dimitrov K. Y Forbig J. (2004) O noua strategie euro-atlantica pentm regiunea Marii Negre. Bucarest, Institutul Român de Studii Internationale Nicolae Titulescu.

Badescu, I. y Dungaciu, D. (2002) Sociologia si politica frontierei. Bucarest, Ed. Universitatii. 
Baltac, G. (2003) Ce se intîmpla în Delta Dunarit? Bucarest, Ed. Ziua.

Basescu, T. (2005) "Marea Neagra - promovarea libertatii, a democratiei si a stabilitatii regionale". Discurso pronunciado por el Presidente de Rumanía, Traian Basescu en Council of Foreign Relations el 10 de marzo de 2005 en Washington, y publicado por el diario rumano ZIUA el 11 de marzo de 2005 www.ziua.ro

Berg, E. (1994) "Un nouvel ordre mundial" Défense Nationale, fevrier, 1994.

Bloch-Laine, A. (1996) "Le binôme sécurité -défense dans L'Europe de l'après guerre froide". Défense Nationale, 1996.

Brzezinski, Z. (1989) The Gran failure: The Birth and Decay of Communism in the Twentieth Century. New York, Charles Schribner's.

Chirot, D y Jowitt, K., (1987) Beginning E.E.P.S, Eastern European Politics and Societies, 2, 12-15.

Damian G. (2005) "2005: 65 de ani de la invadarea României de catre URSS". Ziua, 28 de junio de 2005.

Delorme, R. (1996) A L'est du nouveau changement institutionnel et transformations économiques. Paris Editiones l'Harmattan.

Ionescu, C. (2005) Marea Neagra - un pivot geopolitic in disputa? Revista de Geopolitica Regionala, 1 (5) 2005, an IV, Universitatea Bucarest.

Iorga, N (1936) Istoria poporului român. Bucarest, Ed. Meridiane.

Iovcev, 1. (2005) Genocid cultural Ziua, 10 de agosto de 2005.

Jackson, P. B. (2005) American Strategy for the Black Sea region. 10 de marzo de 2005 www.ziua.ro

Kjellen, R. (1920) Grundniss zu einem System der Politik. Leipzig 64-65.

Mackinder, H. (1919) Democratic Ideals and Reality. Geograpbical Journal, New York, $47-48$.
Mackinder, H. (1919) Geographical Pivot of History. Geograpbical Joumal, New York, 78-79.

Marcu, S. (2006) Un puente latino sobre Europa. Las relaciones Rumania-España en el nuevo contexto europeo. Bucarest, Editorial del Instituto Cultural Rumano.

Marakuta, G. (2005) Niciodata nu ne vom inclina in fata steagului românesc. Rusia la $z i, 20$ de noviembre de 2005.

Menar, S. (2005) Conflictos latentes en la región del mar Negro. Bucarest, Ziua

Nouzille, J. (2005) Moldova. Istoria tragica a unei regiuni europene, Chisinau, Prut Internacional.

Padureanu, D. (2005) Insula Serpilor. Bucarest, Ziua.

Parvan, V. (1923) Inceputurile vietii romane la gurile Dunarii. Bucarest, Lumea.

Pavliuk O. y Klympush-Tsintsadze, I. (2004) The Black Sea Region. Cooperation and Security Building. New York East West Institute.

Plaza Gutiérrez, J. (2000) Geografia de Europa. López Palomeque, F. coord. Cap. 15. Desencuentros, conflictos y confrontaciones: fracturas sociales y bélicas en Europa, 445-462. Barcelona, Ariel Geografía.

Roncea V. coord. (2005) Axa - Noua Romanie la Marea Neagra. Bucarest, Ziua.

Roncea V. (2004) Romania în noua ordine mondiala. Bucarest, Ziua.

Serebrian, O. (1998) Va exploda estul? Geopolitica spatiului pontic. Cluj Napoca, Dacia.

Serebrian, O. (2004) Politica si geopolitica Bucarest, Cartier.

Simileanu, V. (2003) România. Tensiuni geopolitice. Bucarest, Top Forum.

Tismaneanu, V. (1999) Revolutille din 1989 Iasi, Polirom. 